M. Stöhr, R. Sadanandan and W. Meier, Phase-resolved characterization of vortex-flame interaction in a turbulent swirl flame, Experiments in Fluids 51 (2011) 1153-1167.

The original publication is available at www.springerlink.com

http://dx.doi.org/10.1007/s00348-011-1134-y 


\title{
Phase-resolved characterization of vortex-flame interaction in a turbulent swirl flame
}

\author{
M. Stöhr • R. Sadanandan • W. Meier
}

Received: date / Accepted: date

\begin{abstract}
The relation between flow field and flame structure of a turbulent swirl flame is investigated using simultaneous particle image velocimetry (PIV) and planar laser-induced fluorescence of $\mathrm{OH}$ (OH-PLIF). The measurements are performed in one axial and three transverse sections through the combustion chamber of a gas turbine model combustor, which is operated with methane and air under atmospheric pressure. Analysis of the velocity fields using proper orthogonal decomposition (POD) shows that the dominant unsteady flow structure is a so-called precessing vortex core (PVC). In each of the four sections, the PVC is represented by a characteristic pair of POD eigenmodes, and the phase angle of the precession can be determined for each instantaneous velocity field from its projection on this pair. Phase-conditioned averages of velocity field and $\mathrm{OH}$ distribution are thereby obtained and reveal a pronounced effect of the PVC in the form of convectionenhanced mixing. The increased mixing causes a rapid ignition of the fresh gas, and the swirling motion of the PVC leads to an enlarged flame surface due to flame roll-up. A three-dimensional representation shows that the PVC is accompanied by a co-precessing vortex in the outer shear layer, which, however, has no direct impact on the flame. As an alternative to phase averaging, a low-order representation of the phase-resolved dynamics is calculated based on the first pair of POD modes. It is found that small-scale structures are represented more accurately in the phase averages, whereas
\end{abstract}

M. Stöhr · R. Sadanandan · W. Meier

Institute of Combustion Technology,

German Aerospace Center (DLR),

Pfaffenwaldring 38-40,

70569 Stuttgart, Germany

E-mail: michael.stoehr@dlr.de the low-order model has a considerable smoothing effect and therefore provides less detailed information. The findings demonstrate that the combined application of POD, PIV and PLIF can provide detailed insights into flow-flame interaction in turbulent flames.

\section{Introduction}

The interaction of flow field and flame plays an essential role in gas turbine (GT) combustion. Critical phenomena like flame stabilization mechanisms, thermoacoustic oscillations or lean blowout involve complex interactions of unsteady flow structures and chemistry, which are still not well enough understood today. The currently desired improvements of GT combustors with respect to emissions, fuel flexibility and reliability therefore depend considerably on a better understanding of these interactions.

Unsteady flow structures, including both irregular turbulent motions and quasi-periodic dynamics of coherent flow structures, can interact with a flame in various ways as reviewed by Renard et al. (2000). E.g., the flow can influence the flame through transport and mixing, leading to changes of local gas composition and temperature. Furthermore, vortical structures may induce an enlarged flame surface or lead to strain-induced local extinction (Renard et al. 1999). The magnitude of each effect strongly depends on various parameters like, e.g., local gas composition and temperature, relative orientation of flame front and velocities, and time scales of chemistry and flow. Conversely, the flame affects the flow field due to the expansion induced by heat release, and leads to changes of flow parameters such as density or viscosity. For highly turbulent GT-typical flames, where several effects may compete and inter- 
act, accurate modeling of these phenomena is almost impossible, and therefore further detailed experimental studies are required.

A rewarding experimental approach for studying flow-flame interaction is the simultaneous co-planar measurement of velocity field and flame structure using minimally-intrusive laser-based techniques (Meier et al. 2010). Typically a combination of particle image velocimetry (PIV) and planar laser-induced fluorescence (PLIF) is applied. Using this approach, flow-flame interaction has been studied in counterflow diffusion flames (Lemaire et al. 2004; Meyer et al. 2004), lifted diffusion flames (Watson et al. 2002), propagating flat flames (Mueller et al. 1995, 1998), swirl flames (Tanahashi et al. 2005; Wicksall et al. 2005; Petersson et al. 2007; Sadanandan et al. 2008; Stöhr et al. 2009) and turbulent jet flames (Rehm and Clemens 1998; Kothnur et al. 2002; Hult et al. 2005). More recently, high-speed lasers and cameras with (multi-) $\mathrm{kHz}$ repetition rates have been used for time-resolved simultaneous PIV and OH-PLIF measurements of transient phenomena such as lean blowout (Chaudhuri et al. 2010, 2011; Stöhr et al. 2011), local extinction (Böhm et al. 2009; Boxx et al. 2010), thermoacoustic oscillations (Boxx et al. 2010; Steinberg et al. 2010) and flashback (Konle et al. 2008; Konle and Sattelmayer 2009).

In order to determine conclusive correlations between dynamics of the flow and the flame, it is desirable to have available appropriate statistical tools for analyzing the PIV and PLIF data. This is not trivial because the structures of interest are usually superimposed by turbulence or other unsteady phenomena. In the field of fluid mechanics, the statistical analysis of flow fields using proper orthogonal decomposition (POD) has been established as a valuable tool for the extraction and characterization of coherent vortex structures (Berkooz et al. 1993). In the case of periodic vortex motion, a particular feature of POD is its ability to determine the phase with respect to the periodic cycle. This allows the estimation of conditional averages from non-conditionally recorded data and has been employed, e.g., for phase-resolved characterizations of vortex shedding in cylinder wakes (van Oudheusden et al. 2005; Perrin et al. 2007). It is further noted that the POD-based analyses can be straightforward extended to a combined analysis of synchronized measurements with different techniques (Borée 2003). In combination with simultaneous PIV and PLIF measurements, POD therefore offers great potential for investigations of flowflame interaction. Until now, however, only two studies have made use of this approach, focusing on a laminar flame (Duwig and Iudiciani 2010) and on thermoacoustic oscillations (Steinberg et al. 2010).
The aim of this work is to investigate the role of coherent vortex structures in the stabilization mechanism of a GT-typical turbulent swirl flame by means of simultaneous PIV and OH-PLIF measurements. A particular focus is to explore and utilize the capabilities of a PODbased analysis of such combined measurements with regard to vortex-flame interaction. The study employs a GT model combustor, which at certain operating conditions exhibits thermoacoustic oscillations. These oscillations have been subject of numerous previous experimental studies, where several diagnostic techniques have been applied such as Raman spectroscopy, PLIF and PIV at repetition rates between $5 \mathrm{~Hz}$ and $5 \mathrm{kHz}$. Recent reviews of these works are provided by Boxx et al. (2010) and Steinberg et al. (2010). In another work, where the same combustor was operated at a fuel-lean condition, Stöhr et al. (2011) have investigated the dynamics of lean blowout.

For the present study, the combustor is operated at a condition where no blowout and virtually no thermoacoustic oscillations occur, but a pronounced hydrodynamic instability in the form of a PVC is present. PVCs are often encountered in swirl flames, but their interaction with the flame is still not well understood (Syred 2006). The work employs OH-PLIF and PIV at a low repetition rate $(5 \mathrm{~Hz})$, which provides a large field of view (ca. $80 \times 60 \mathrm{~mm}^{2}$, compared to $30 \times 30 \mathrm{~mm}^{2}$ for PIV at $5 \mathrm{kHz}$ ) across the whole width of the combustion chamber and thus allows a more comprehensive analysis of coherent flow structures. The measurements are performed in one axial and three transverse sections through the combustion chamber. In the PODbased analysis the PVC appears in each section as a pair of eigenmodes. The corresponding POD mode coefficients are used to determine the phase angle, and thereby phase averages of velocity and $\mathrm{OH}$ are calculated. Based on these results the effect of the PVC on mixing, ignition and thus on the shape of the flame zone is examined. Phase averages are also used to determine the three-dimensional structure of the PVC. Using an extended POD, a low-order model of the dynamics associated with the PVC is calculated and compared to the phase averages.

\section{Experimental setup}

\subsection{Combustor and operating condition}

The layout of the GT model combustor is shown in Fig. 1. Dry air first enters a plenum (diameter $79 \mathrm{~mm}$, height $65 \mathrm{~mm}$ ) and then separately passes two radial swirl generators. The two co-swirling flows enter the combustion chamber through a central circular nozzle 


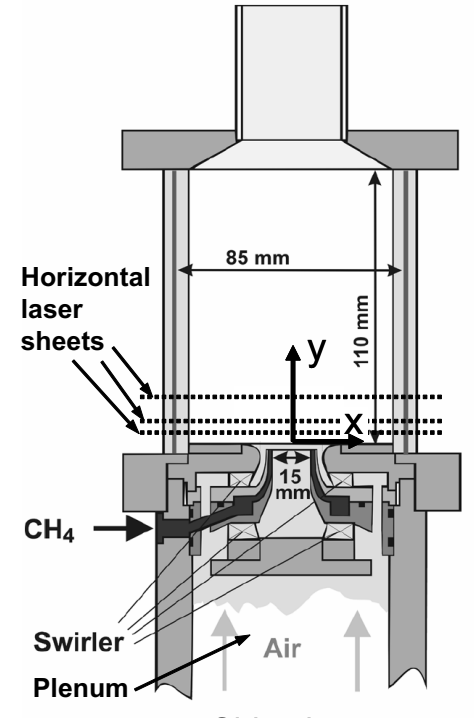

\section{a}

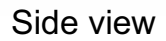

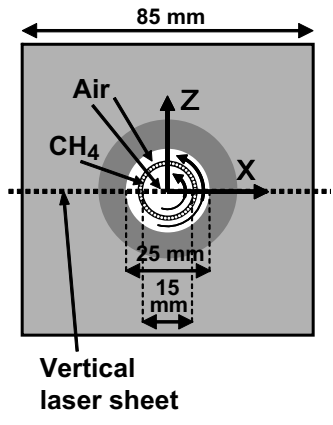

Top view of b burner nozzle

Fig. 1 (a) Side view of the combustor and (b) sketch of burner exit plane. The curved arrows indicate the sense of swirl.

(diameter $15 \mathrm{~mm}$ ) and a surrounding annular nozzle (inner diameter $17 \mathrm{~mm}$, outer diameter $25 \mathrm{~mm}$ contoured to an outer diameter of $40 \mathrm{~mm})$. Non-swirling $\mathrm{CH}_{4}$ is supplied through 72 channels $\left(0.5 \times 0.5 \mathrm{~mm}^{2}\right)$ forming a ring between the air nozzles. The exit planes of the fuel and central air nozzles are located $4.5 \mathrm{~mm}$ below the exit plane of the outer air nozzle which is taken as height $y=0$. The combustion chamber has a square cross-section of $85 \times 85 \mathrm{~mm}^{2}$, a height of $114 \mathrm{~mm}$, and is enclosed by four quartz windows held by four posts (diameter $10 \mathrm{~mm}$ ) in the corners allowing very good optical access. A conical top plate with a central exhaust tube (diameter $40 \mathrm{~mm}$, length $50 \mathrm{~mm}$ ) forms the exit. In the present study the flame was operated at room temperature and atmospheric pressure with a thermal power $\mathrm{P}_{\text {thermal }}=8.4 \mathrm{~kW}$ and a global equivalence ratio $\phi=0.88$. The corresponding Reynolds number of the cold inflow is $\operatorname{Re} \approx 9,000$. From velocity profiles at $y=1$ $\mathrm{mm}$ the swirl number, calculated as the ratio between the tangential and axial momentum flux, has been estimated in a previous work for similar operating conditions as $S \approx 0.55$ (Weigand et al. 2006).

\subsection{Laser diagnostics}

\subsubsection{Simultaneous PIV and OH-PLIF technique}

The diagnostic setup consists of two separate laser and camera systems used for simultaneous PIV and $\mathrm{OH}-$ PLIF measurements, which are separately described be-

low. The light sheets from the two laser systems are overlapped into a common plane crossing the combustion chamber. The setup was used in two versions with different orientations of the light sheets: first, a vertical section through the central axis $(z=0$, shown in Fig. $1 b)$, and second, horizontal transverse sections located at $y=5,10$ and $20 \mathrm{~mm}$ (shown in Fig. 1a). In the former case, two-component velocity vectors were obtained using a single PIV camera. For the latter configuration, the PIV system was extended to a stereoscopic setup as shown in Fig. 2, where both the two PIV cameras and the LIF camera are located diagonally above the laser sheet. For each of the four sections, a series of 1500 measurements was performed with a field of view of ca. $80 \times 60 \mathrm{~mm}^{2}$ and an acquisition rate of $5 \mathrm{~Hz}$. In addition, $\mathrm{OH}^{*}$-chemiluminescence (integrated along the line-of-sight) was measured using the OH-PLIF detection system for the vertical section.

\subsubsection{OH-PLIF system}

The fluorescence of $\mathrm{OH}$ was excited by means of a frequency-doubled flashlamp-pumped Nd:YAG laser (Spectra-Physics PIV-400-10) pumping a frequencydoubled tuneable dye laser (Sirah PRSC-G-24-EG). The UV laser pulse had a duration of $7 \mathrm{~ns}$, a line width of $0.4 \mathrm{~cm}^{-1}$ and a pulse energy of approximately $2.5 \mathrm{~mJ}$. It was tuned to the $\mathrm{Q}_{1}(8)$ transition of $\mathrm{OH}$ at approximately $283 \mathrm{~nm}$ within the vibrational band $\nu^{\prime \prime}=0, \nu^{\prime}=1$ of the $A^{2} \Sigma^{+}-X^{2} \Pi$ system. The population of the lasercoupled ground state of the selected line varied by approximately $9 \%$ in the temperature range between 1400 $\mathrm{K}$ and $2200 \mathrm{~K}$. A small portion of the laser beam was deflected by a beamsplitter into a methane-air reference flame stabilized on a McKenna type burner, operated under laminar conditions. The LIF signal produced by the flame was used for online monitoring of the excitation wavelength. Through a combination of two cylindrical lenses, the beam was then expanded to a planar sheet with a height of ca. $60 \mathrm{~mm}$ and a thickness of $\approx 400 \mu \mathrm{m}$ inside the combustor.

OH fluorescence in the $\nu^{\prime}=1, \nu^{\prime \prime}=1$ and $\nu^{\prime}=0, \nu \nu^{\prime \prime}=0$ bands near $310 \mathrm{~nm}$ was detected by a CCD camera (LaVision Imager Intense, $1376 \times 1040$ pixel) with image intensifier (LaVision IRO) equipped with a Halle UV lens (focal length $f=64 \mathrm{~mm}, f / 2.0$ ) and a bandpass filter (transmission range $300-325 \mathrm{~nm}$ ). The filter suppressed background radiation and elastically scattered laser light from the OH-PLIF and PIV systems. Before the combustor, part of the incoming laser sheet was deflected by a beam splitter into a quartz cell filled with fluorescent dye solution. The dye cell fluorescence profile was simultaneously imaged using another CCD 
camera with image intensifier (LaVision Imager Intense with IRO) equipped with a Nikon lens $(f=50 \mathrm{~mm}$, $f / 4.0)$. This dye cell profile is used for correcting shotto-shot laser sheet profile inhomogeneities. The image intensifier was set to an exposure time of $400 \mathrm{~ns}$ for both $\mathrm{OH}-\mathrm{PLIF}$ and dye cell fluorescence cameras. The spatial resolution of the system based on the size of a pixel in the combustor is approximately $100 \mu \mathrm{m}$. In order to perform synchronized measurements, a trigger signal from the PTU of the PIV system was distributed to the lasers and cameras of the PLIF system via a multi-channel pulse delay generator (BNC Model 565). The laser flashlamps were fired at $10 \mathrm{~Hz}$, whereas the Q-switches and cameras were triggered at $5 \mathrm{~Hz}$. The PLIF image was captured between the two PIV images at $10 \mu$ s after the first PIV laser pulse.

\subsubsection{PIV system}

The PIV system (LaVision Flowmaster) consisted of a frequency-doubled dual-cavity Nd:YAG laser (NewWave Solo PIV 120), a double-frame CCD camera (LaVision Imager intense, $1376 \times 1040$ pixels) and a programmable timing unit (PTU). The laser pulses had a duration of $4 \mathrm{~ns}$ and an energy of $120 \mathrm{~mJ}$ at $532 \mathrm{~nm}$. After passing through sheet forming optics, the laser beam had a height of approximately $60 \mathrm{~mm}$ and a thickness of $1 \mathrm{~mm}$ inside the combustor. The time separation between the two laser pulses was 20 s. The CCD camera was equipped with a Nikon lens $(f=50 \mathrm{~mm}, f / 5.6)$ covered with a narrow bandpass filter $(532 \pm 5 \mathrm{~nm})$ for reducing the influence of flame luminosity. For stereoscopic PIV a second identical CCD camera was installed, and both cameras were mounted on Scheimpflug adaptors in order to align the focal plane with the plane of the laser sheet. Laser and camera(s) were triggered by the PTU at a repetition rate of $5 \mathrm{~Hz}$. The air flow was seeded with $\mathrm{TiO}_{2}$ particles with a diameter of $1 \mu \mathrm{m}$.

Velocity fields were estimated from the PIV images using a commercial PIV software (LaVision DaVis 7). A multi-scale cross-correlation algorithm was used, with the interrogation window size ranging from $32 \times 32$ pixel to $16 \times 16$ pixel. In the hot gas regions, approximately six particles were present in an area of $16 \times 16$ pixel, whereas in the unburned gas, the density was considerably higher (more than 12 particles per interrogation area). Spurious vectors were detected using the deviation from the local median, and replaced either by vectors corresponding to the $2 \mathrm{nd}$, 3rd or 4 th highest correlation peak (if they fulfil the median criterion), or else by an interpolated vector. The fraction of interpolated vectors was less than $1 \%$. The spatial resolution of

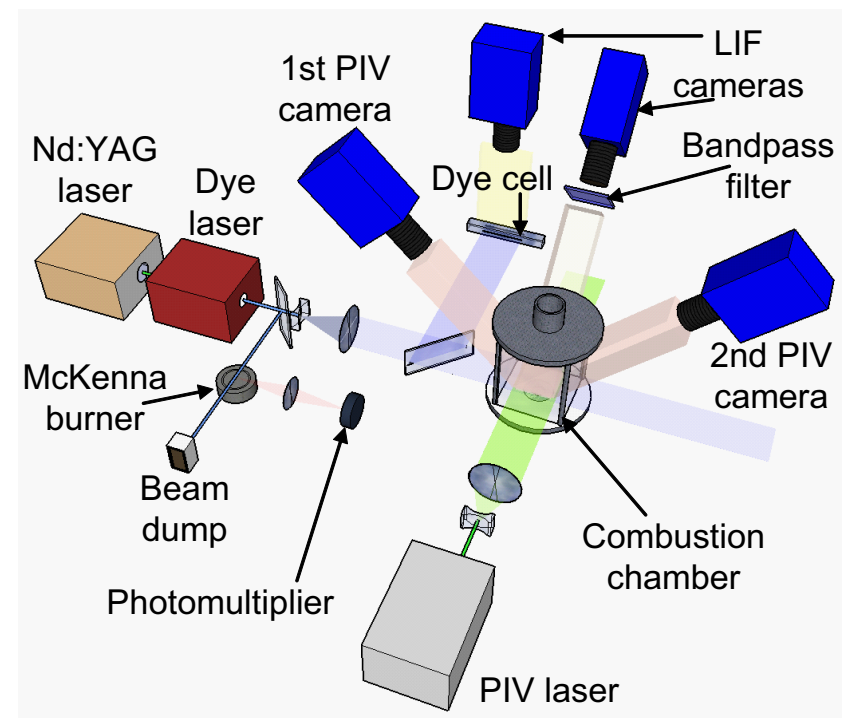

Fig. 2 Setup for simultaneous PIV and OH-PLIF measurements.

velocities is $1.1 \mathrm{~mm}$ (corresponding to an interrogation window of $16 \times 16$ pixels). Based on the 0.1 pixel uncertainty of the cross-correlation peak-finding algorithm, the uncertainty of the velocities is estimated to be 0.3 $\mathrm{m} / \mathrm{s}$. An additional perspective error, resulting from the out-of-plane velocity, can occur in the two-component velocity fields measured in the vertical section. This error is maximal in the region of strongly swirling flow above the nozzle, where it was estimated to induce a relative uncertainty of up to $4 \%$ for axial and $6 \%$ for radial velocities.

\section{Proper Orthogonal Decomposition}

\subsection{Basic principles}

Proper orthogonal decomposition (POD) is a statistical tool that has been established in the field of fluid mechanics as a method for the extraction and characterization of unsteady coherent flow structures. The following description introduces the most important principles that are relevant for this work, while details are provided in the review by Berkooz et al. (1993).

Starting point is a series of $N$ velocity vector fields $\vec{u}_{i}\left(\vec{x}_{k}\right)(i=1 . . N)$ measured at $M$ positions $\vec{x}_{k}(k=$ 1..M). At first, the velocity fields are decomposed into an average $\overline{\vec{u}}$ and a fluctuating part $\vec{u}^{\prime}$ :

$\vec{u}_{i}\left(\vec{x}_{k}\right)=\overline{\vec{u}}\left(\vec{x}_{k}\right)+\vec{u}_{i}^{\prime}\left(\vec{x}_{k}\right)$

The principal idea behind POD is then to determine a set of linear basis functions, also called POD modes, which optimally represent the fluctuating part $\vec{u}_{i}^{\prime}$ of the 
flow. More precisely, the POD modes $\vec{\phi}_{j}\left(\vec{x}_{k}\right)(j=1 . . N)$ are chosen such that they have the following properties:

(i) The modes $\vec{\phi}_{i}$ form an orthonormal basis,

$<\vec{\phi}_{i} \cdot \vec{\phi}_{j}>=\delta_{i j}$

The angled brackets here denote spatial integration, and $\delta_{i j}$ is the Kronecker delta. The velocity fields $\vec{u}_{i}$ can then be expanded to any order $R(1 \leq R \leq N)$ relative to the modes $\vec{\phi}_{j}$ :

$\vec{u}_{i}^{(R)}\left(\vec{x}_{k}\right)=\overline{\vec{u}}\left(\vec{x}_{k}\right)+\sum_{j=1}^{R} a_{i j} \vec{\phi}_{j}\left(\vec{x}_{k}\right)$

The mode coefficients $a_{i j}$ denote the projection of the velocity field $\vec{u}_{i}^{\prime}$ on mode $\vec{\phi}_{j}$,

$a_{i j}=<\vec{u}_{i}^{\prime} \cdot \vec{\phi}_{j}>$.

(ii) Each mode $\vec{\phi}_{j}$ contributes a specific part $\lambda_{j}$ to the total fluctuating kinetic energy $E_{\text {tot }}$ (calculated omitting the density):

$E_{\mathrm{tot}}=\sum_{i=1}^{N}<\vec{u}_{i}^{\prime} \cdot \vec{u}_{i}^{\prime}>=\sum_{j=1}^{N} \lambda_{j}$

$\lambda_{j}=\frac{1}{N} \sum_{i=1}^{N} a_{i j}^{2}$

The modes are arranged such that their energies are sorted in non-increasing order, i.e., $\lambda_{i} \geq \lambda_{i+1}$.

(iii) For any $R$, the energy

$E^{(R)}=\sum_{j=1}^{R} \lambda_{j}$

contained in the first $R$ modes is higher than for any other orthonormal basis. In other words, the POD is optimal with respect to kinetic energy for low-order representation of the velocity fields $\vec{u}_{i}^{(R)}\left(\vec{x}_{k}\right)$ according to Eq. 3. The first modes thus represent the most dominant unsteady flow structures.

(iv) The POD modes are related to the mode coefficients $a_{i j}$ and the energies $\lambda_{j}$ by

$\vec{\phi}_{j}=\frac{1}{\lambda_{j} N} \sum_{i=1}^{N} a_{i j} \vec{u}_{i}^{\prime}$

\subsection{Method of snapshots}

The determination of POD modes with the above properties leads to an eigenvalue problem for a matrix of size $M \times M$, which will not be derived here. In the original work of Lumley (1967), this eigenvalue problem is directly solved. Modern PIV systems, however, typically provide velocity fields with such a large number $M$ of spatial points (in this case $M \approx 10000$ ) that the direct solution is computationally extremely expensive. On the other hand, the number of measurements is often significantly lower (in this case $N=1500$ ). For such situations $(N<M)$, the POD modes can be determined more efficiently and generally in good approximation by the method of snapshots introduced by Sirovich (1987). This method, which is used in the present work, determines the POD modes $\vec{\phi}_{j}$ from the eigenvalues $\lambda_{j}$ and eigenvectors $\vec{e}_{j}=\left[e_{1 j}, e_{2 j}, \ldots, e_{N j}\right]$ of the $N \times N$ correlation matrix $K_{i j}$ :

$K_{i j}=\frac{1}{N}<\vec{u}_{i}^{\prime} \cdot \vec{u}_{j}^{\prime}>$

$K \vec{e}_{j}=\lambda_{j} \vec{e}_{j}$,

The mode coefficients are then given by

$a_{i j}=e_{i j} \sqrt{\lambda_{j} N}$,

and the POD modes are calculated using Eq. 8.

\subsection{Extended analysis}

In addition to the analysis of coherent flow structures, the POD method can also be used to identify correlations between flow structures and other measured quantities like concentration, temperature or pressure. This so-called extended POD analysis has been introduced by Borée (2003).

In the following, a set of concentration fields $c_{i}\left(\vec{x}_{l}\right)$ $(i=1 . . N)$ measured at positions $\vec{x}_{l}\left(l=1 . . M_{c}\right)$ is considered, which have been measured simultaneously with the velocity fields $\vec{u}_{i}\left(\vec{x}_{k}\right)$. The $c_{i}$ are first decomposed into an average $\bar{c}$ and a fluctuating part $c_{i}^{\prime}$ :

$c_{i}\left(\vec{x}_{l}\right)=\bar{c}\left(\vec{x}_{l}\right)+c_{i}^{\prime}\left(\vec{x}_{l}\right)$

Analogous to Eq. 8, a set of extended POD modes $\chi_{j}\left(\vec{x}_{l}\right)$ is now defined as

$\chi_{j}=\frac{1}{\lambda_{j} N} \sum_{i=1}^{N} a_{i j} c_{i}^{\prime}$,

where the mode coefficients $a_{i j}$ are obtained from the POD of velocity fields as described in Sect. 3.2. The extended POD modes are the key quantity for determining the correlation between coherent flow structures and the concentration fields. It can be shown (Borée 2003) that the part of the concentration field $c_{i}^{\prime}$ that is correlated with the velocity POD mode $\vec{\phi}_{j}$ is given by $a_{i j} \chi_{j}$. For any given range of velocity POD modes $\vec{\phi}_{j}$ $(j=1 . . R)$, each concentration field $c_{i}^{\prime}$ can then be decomposed into its part that is correlated to these modes,

$c_{i, \mathrm{corr}}^{\prime}\left(\vec{x}_{l}\right)=\sum_{j=1}^{R} a_{i j} \chi_{j}\left(\vec{x}_{l}\right)$, 

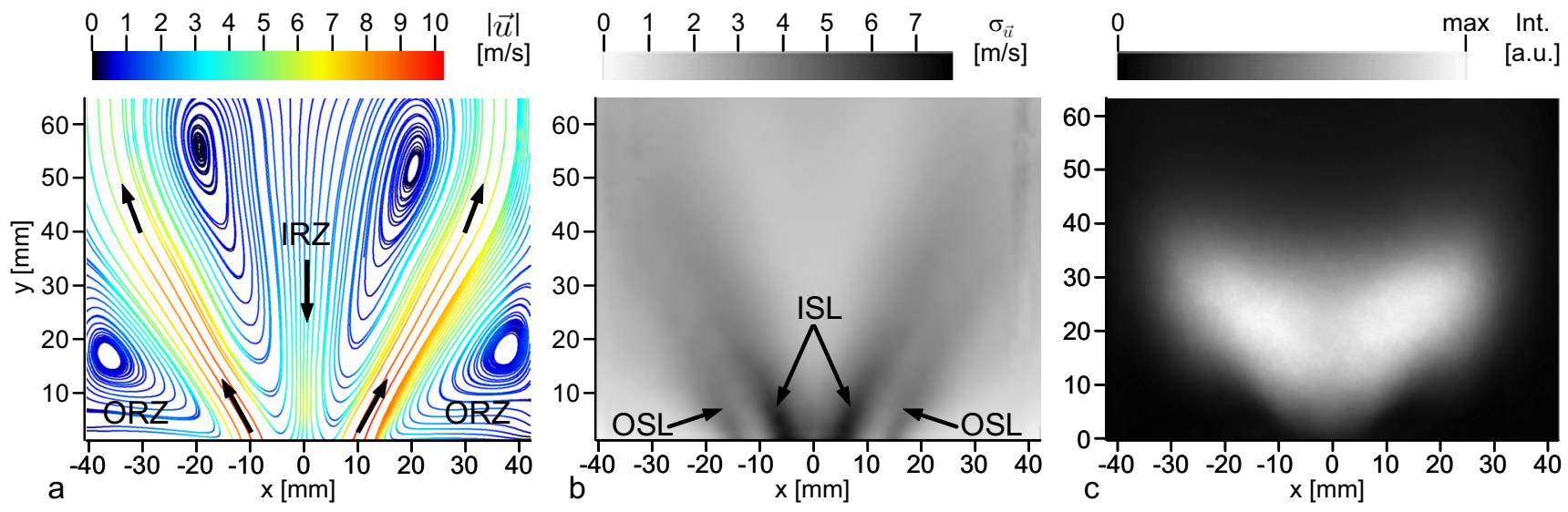

Fig. 3 (a) Average and (b) RMS values of velocity field; (c) average $\mathrm{OH}$ chemiluminescence.

and the part that is not correlated to these modes,

$c_{i, \text { uncorr }}^{\prime}\left(\vec{x}_{l}\right)=c_{i}^{\prime}\left(\vec{x}_{l}\right)-c_{i, \text { corr }}^{\prime}\left(\vec{x}_{l}\right)$

\section{Average and instantaneous results}

\subsection{Average velocity field and flame shape}

The average and RMS values of the velocity field are shown in Fig. 3a and b, respectively. The average flow field is typical of confined swirl flames. Vortex breakdown of the swirling air flow occurs shortly below the nozzle exit plane $(y=0 \mathrm{~mm})$ and leads to a cone-shaped stream of fresh gas into the chamber and to the formation of a large inner recirculation zone (IRZ). Furthermore an outer recirculation zone (ORZ) is formed due to the steplike cross-section extension at the nozzle exit. High RMS velocities occur in the regions of strong velocity gradients, namely the inner shear layer (ISL) between the inflow and the IRZ, and the outer shear layer (OSL) between inflow and ORZ. The average $\mathrm{OH}^{*}$ signal (integrated along line-of-sight) shown in Fig. 3c is used as a marker of the location of heat release (Hardalupas and Orain 2004). It can be seen that the heat release zone starts at $y \approx 3 \mathrm{~mm}$ and extends up to $y=40 \mathrm{~mm}$ within a radius of $r<35 \mathrm{~mm}$ from the centerline. The flame base therefore lies approximately $7 \mathrm{~mm}$ above the fuel nozzle exit, which indicates that a high level of mixing has occured before ignition and that the flame is of partially premixed nature.

\subsection{Instantaneous fields of velocity and $\mathrm{OH}$}

A streamline plot of an instantaneous PIV measurement in the vertical section is shown in Fig. 4a. The flow field exhibits a zig-zag arrangement of vortices in the ISL marked by black arrows. Such a zig-zag arrangement, which appears with varying axial displacements in most other instantaneous measurements in the vertical section (not shown), indicates the presence of a coherent 3D helical vortex in the ISL. This type of vortex is often found in swirl flames (Syred 2006) and is commonly termed a precessing vortex core (PVC). Furthermore some more or less strong vortices appear in the OSL (marked by gray arrows) at positions that are obviously associated with the vortices in the ISL. From pressure recordings in the combustion chamber, the frequency of the PVC has been estimated as $\approx 360 \mathrm{~Hz}$. The present PIV and OH-PLIF measurements, which were recorded at a repetition rate of $5 \mathrm{~Hz}$, therefore cannot temporally resolve the dynamics of the PVC.

Figure $4 \mathrm{~b}$ shows a vector plot of the same velocity field together with the corresponding OH-PLIF measurement. The grayscale OH-PLIF image characterizes the structure of the flame. Regions without $\mathrm{OH}$ (black) represent gas with low or medium temperature $(T<1500 \mathrm{~K})$, i.e., unburned gas with possible admixture of burned gas. Regions with high levels of $\mathrm{OH}$ (light gray to white) indicate superequilibrium $\mathrm{OH}$, which is formed in the reaction zones and has a half-life period of $\approx 1 \mathrm{~ms}$ under atmospheric pressure (Sadanandan et al. 2008). Medium and low levels of $\mathrm{OH}$ (medium and dark gray) represent burned gas whose $\mathrm{OH}$ concentration has decayed towards equilibrium while it was transported away from the reaction zone. The equilibrium $\mathrm{OH}$ concentration increases with temperature and as the stoichiometric condition is approached (Sadanandan et al. 2008).

In Fig. 4b it can be seen that in the regions around vortices 1 and 2, both unburned gas entering from the nozzle below and hot burned gas coming from the IRZ are present. The spiral-like structures of $\mathrm{OH}$ in these regions show that the vortices lead to an enlarged 

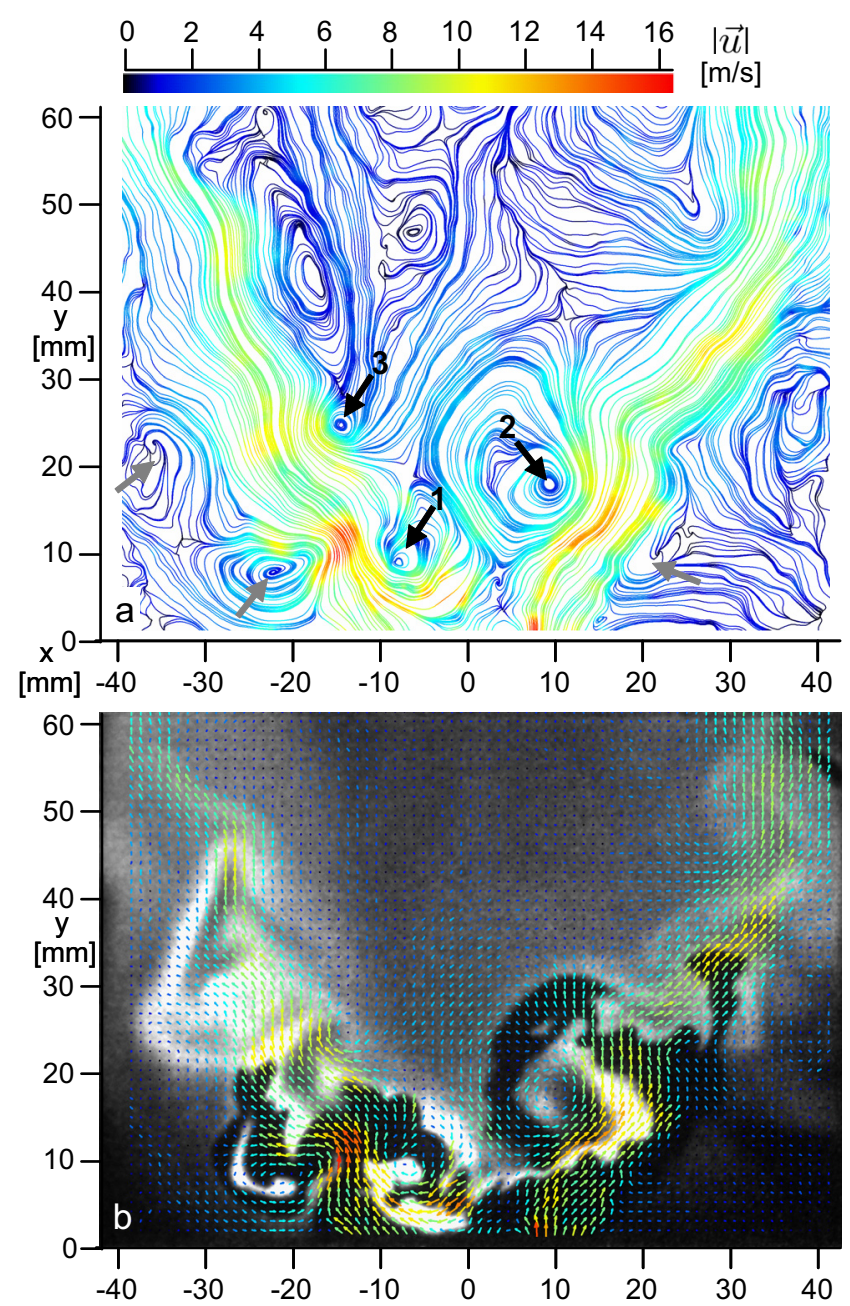

Fig. 4 Simultaneous PIV and OH-PLIF measurement in the vertical section: (a) Streamline plot of the velocity field, (b) Vector plot with corresponding distribution of $\mathrm{OH}$.

boundary and thus to strong convective mixing between burned and unburned gas. The admixture of hot burned gas leads to an increase of temperature and a supply of radicals to the fresh gas, and thus facilitates ignition of the fresh gas. The presence of superequilibrium $\mathrm{OH}$ in the regions around vortices 1 and 2 indicates that reaction immediately takes place in the vicinity of these vortices. Obviously, the flame surface is enlarged due to vortex-induced flame roll-up. In the area around vortex 3 further downstream, on the other hand, reaction progress is largely completed.

Simultaneous PIV and OH-PLIF measurements, which were separately performed in the horizontal sections at $y=5,10$ and $20 \mathrm{~mm}$ are shown in Fig. 5. In each velocity field, a single off-centered vortex (marked by an arrow) appears. A POD-based analysis described later in Sect. 5.3 shows that the off-centered vortex is the representation of the helical PVC in the horizontal
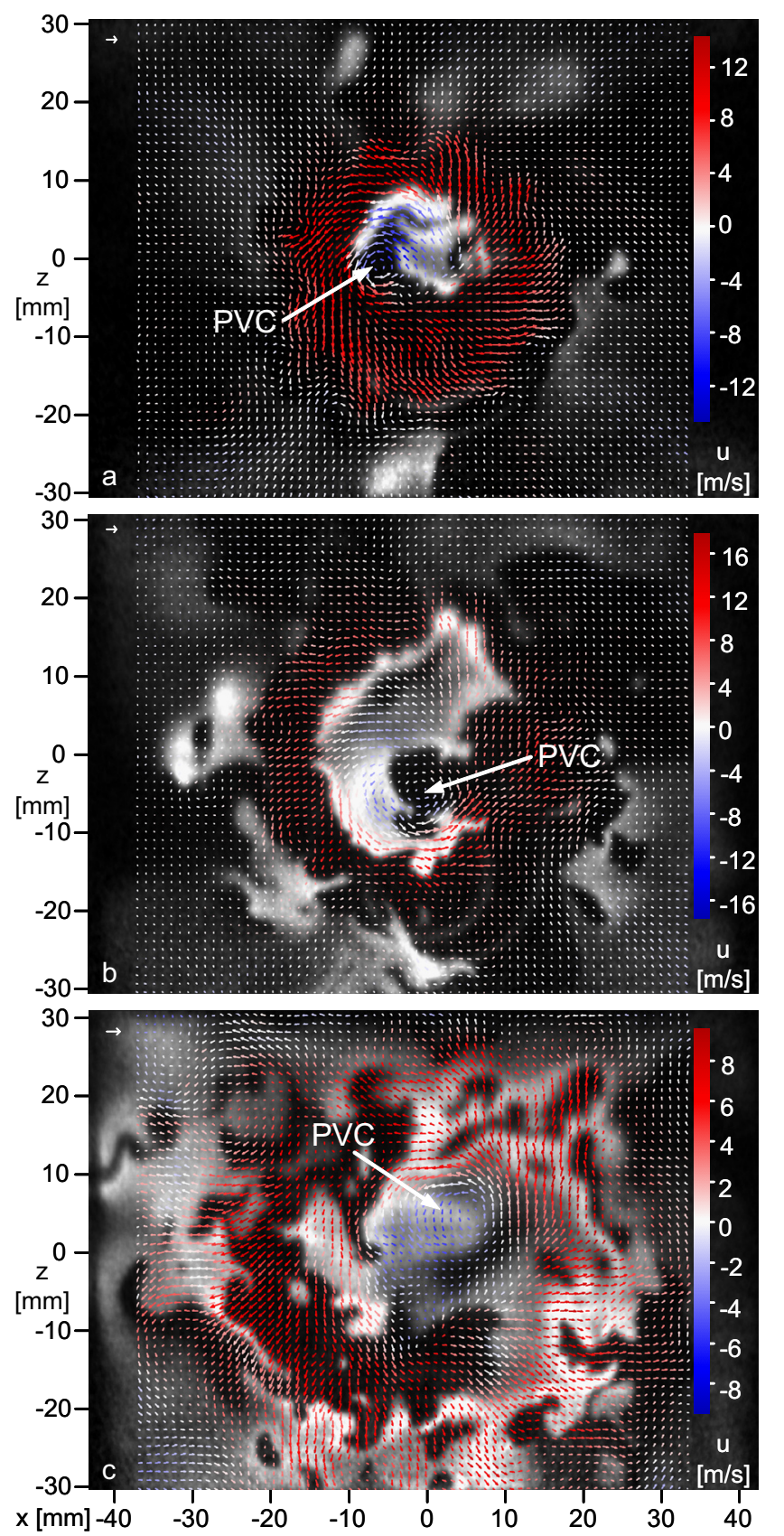

Fig. 5 Simultaneous PIV and OH-PLIF measurements performed separately in horizontal sections at (a) $y=5 \mathrm{~mm}$, (b) $y=10 \mathrm{~mm}$ and (c) $y=20 \mathrm{~mm}$. The arrow in the upper left corners represents $10 \mathrm{~m} / \mathrm{s}$. Vector colors represent the axial velocity.

sections. For $y=5 \mathrm{~mm}$ and $y=10 \mathrm{~mm}$, high $\mathrm{OH}$ levels indicating reaction zones are found in the ISL beside the PVC and are mostly limited to the IRZ. At $y=20$ $\mathrm{mm}$, however, no clear influence of the PVC is found, and reaction takes place in several disconnected zones at larger radial positions $(r>10 \mathrm{~mm})$. Taken together, the instantaneous measurements suggest that the PVC 

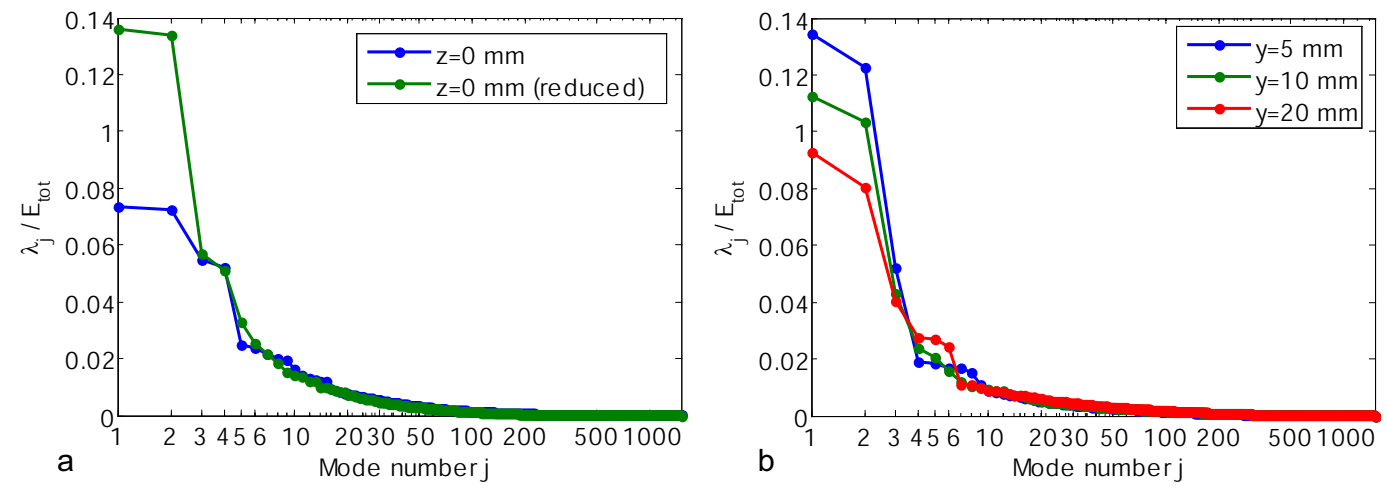

Fig. 7 Eigenvalue spectra for (a) vertical and (b) horizontal sections, representing the contribution of the POD modes to the total fluctuating kinetic energy $E_{\text {tot }}$.
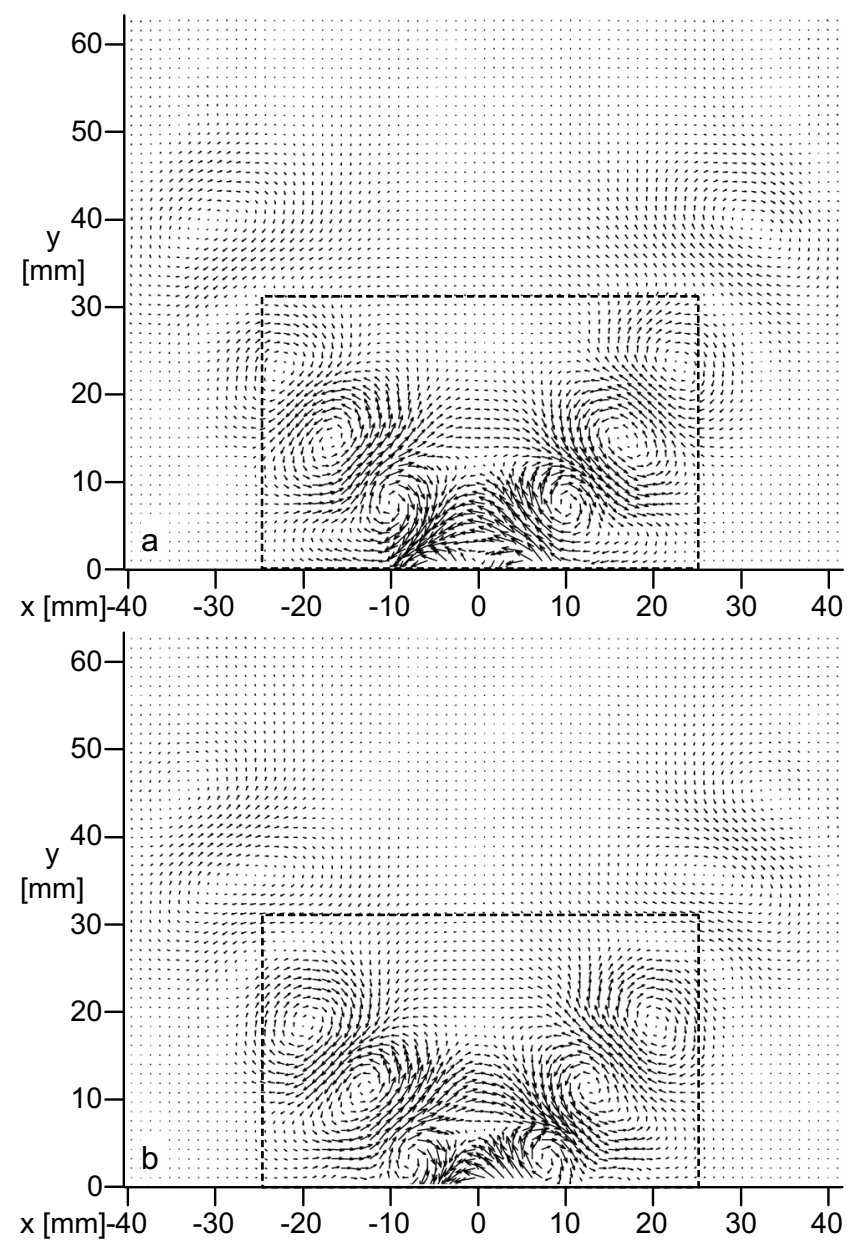

Fig. 6 (a) First and (b) second POD mode of the velocity field in the vertical section. The dotted line indicates the domain of the additional POD that is reduced to the region around the PVC. plays an essential role in the stabilization mechanism of the flame, and that its impact is strongest in the region below $y=20 \mathrm{~mm}$.

\section{POD of the velocity field}

\subsection{Eigenmodes and eigenvalues}

POD analyses of the velocity fields in the vertical and the three horizontal sections have been performed as described in Sect. 3. In all four cases, 1500 instantaneous velocity fields were used. The first two POD modes for the vertical section, which are shown in Fig. 6, are composed of a regular arrangement of vortices in the ISL that represents the PVC. The dual representation of the PVC by two modes is associated with the coherent streamwise motion of vortices due to the precession of the PVC helix. The vortex positions in the two modes are shifted with respect to each other by half of the distance between adjacent vortices. Any intermediate position of the vortices can be represented by an appropriate linear superposition of the two modes. Furthermore it is seen that in the combustion chamber the PVC is strongest at the nozzle exit and extends with decreasing strength to a height of $y \approx 40 \mathrm{~mm}$. The corresponding eigenvalue spectrum plotted in Fig. 7a shows that in the vertical section the PVC contributes $7.4 \%+7.2 \%$ $=14.6 \%$ to the total fluctuating kinetic energy $E_{\text {tot }}$ (neglecting the third velocity component and the density). POD modes 3 and 4 represent another, weaker vortex structure further downstream $(y>30 \mathrm{~mm})$ that will not be discussed in this work. For a more accurate analysis of the mode coefficients (described in section 5.2), a second POD was performed that is restricted to the region of the PVC marked in Fig. 6. As expected, the energy contribution of the $\mathrm{PVC}$ in this region $(13.6 \%$ $+13.4 \%=27 \%$ ) is significantly higher. 

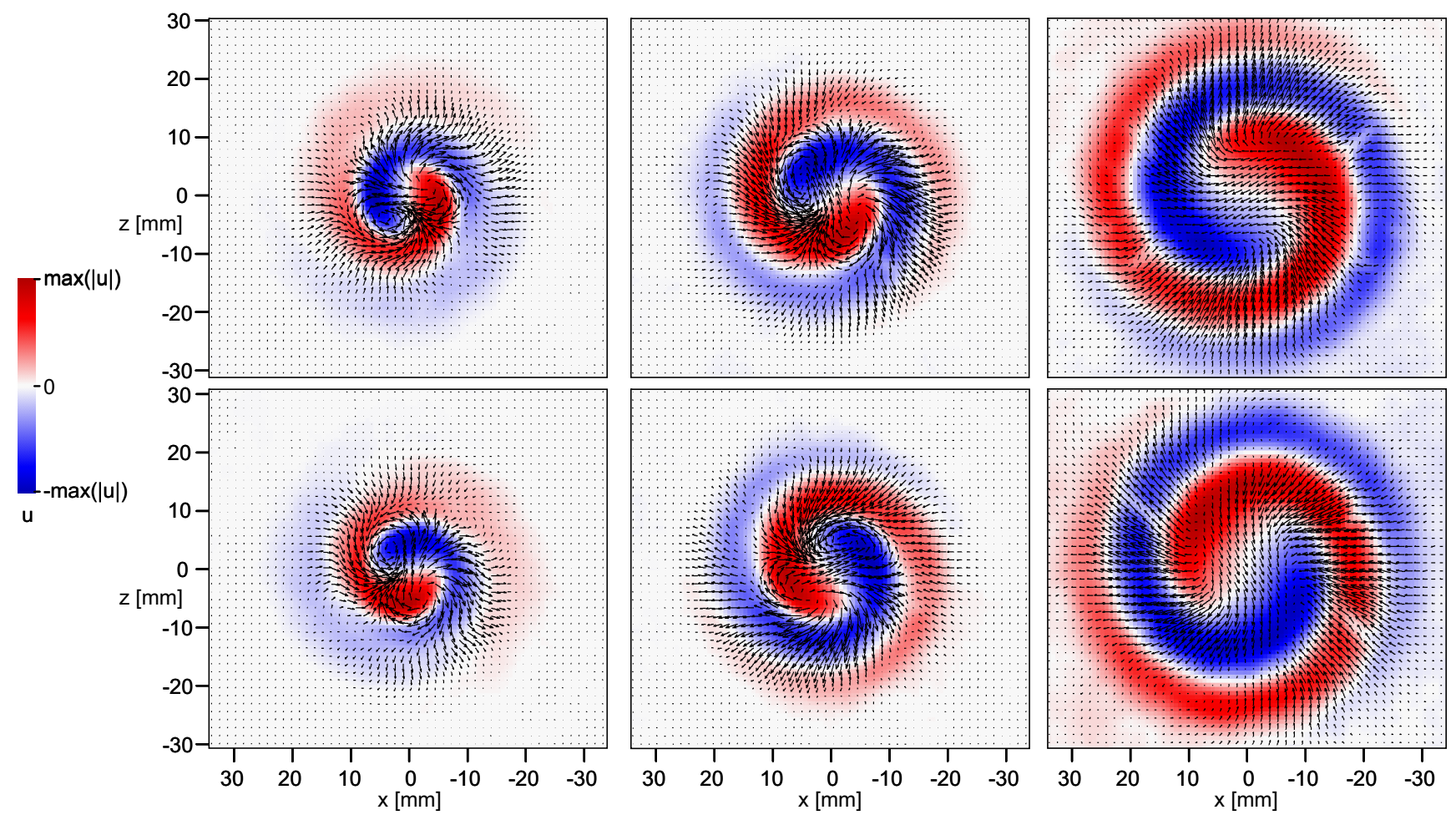

Fig. 8 First (upper row) and second (lower row) POD mode of the velocity field in the horizontal sections at $y=5 \mathrm{~mm}$ (left), $y=10 \mathrm{~mm}$ (middle) and $y=20 \mathrm{~mm}$ (right). Colors represent axial velocity.

Similar to the vertical section, the PVC is represented in the horizontal sections by the first two POD modes. The structures of the modes are shown in Fig. 8 and indicate the increasing radial extension of the vortex in downstream direction. The corresponding energy spectra are plotted in Fig. $7 \mathrm{~b}$. While at $y=5 \mathrm{~mm}$ the PVC adds $13.4 \%+12.3 \%=25.7 \%$ to the energy, the contribution reduces to $11.3 \%+10.3 \%=21.6 \%$ at $y=10$ $\mathrm{mm}$ and $9.3 \%+8.1 \%=17.4 \%$ at $y=20 \mathrm{~mm}$. This behavior corresponds to the reduction of vortex strength observed above for the vertical section. Like in the vertical section, the precession of the PVC can be represented by varying linear superpositions of the first two modes.

\subsection{Determination of phase angle from mode coefficients}

In section 5.1 it was described that the precession of the PVC can be represented by varying linear superpositions of the first two POD modes. This relation is now further analyzed using the mode coeffients $a_{i 1}$ and $a_{i 2}$ of the individual velocity fields. A scatter plot of $a_{i 1}$ vs. $a_{i 2}$ for the vertical section is shown in Fig. 9.

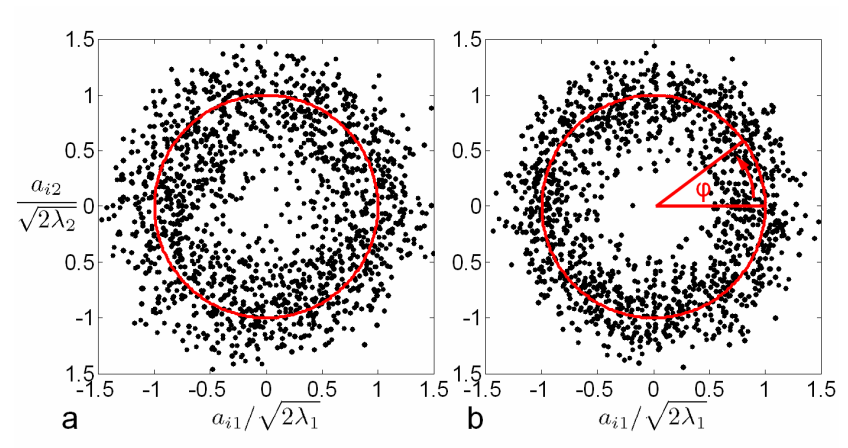

Fig. 9 Scatter plot of mode coefficients $a_{i 1}$ vs. $a_{i 2}$ (a) for the full and (b) for the reduced vertical section.

It can be seen that the coefficients $a_{i 1}$ and $a_{i 2}$ are not independent, but instead are located on the ellipse

$\frac{a_{i 1}^{2}}{2 \lambda_{1}}+\frac{a_{i 2}^{2}}{2 \lambda_{2}}=1$

The observed deviations of the data points from the theoretical curve are caused by turbulent and other unsteady motions that are not necessarily orthogonal to the first two POD modes. As expected these deviations are reduced for the mode coeffients of the second POD that is restricted to the region of the PVC (plotted in Fig. 6). The latter have therefore been used for the following analyses. 


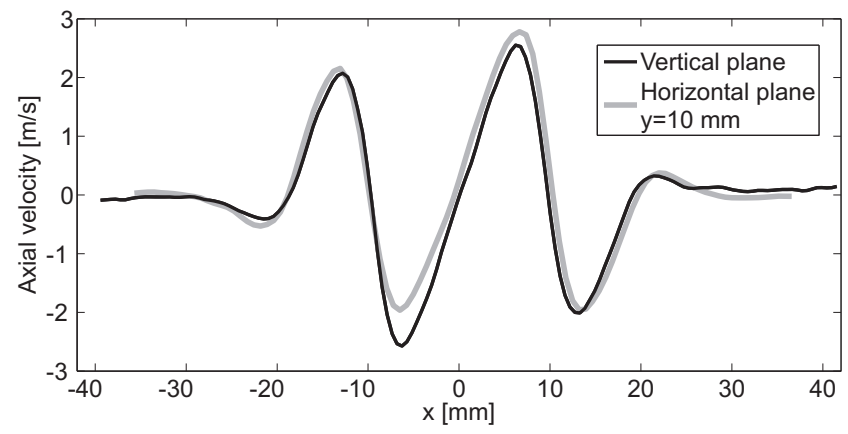

Fig. 10 Comparison of axial velocity profiles at $\varphi=0^{\circ}$ for the POD mode pair of the vertical section (black) and of the horizontal section at $y=10 \mathrm{~mm}$ (gray) along the intersection line $\{y=10 \mathrm{~mm}, z=0 \mathrm{~mm}\}$.

Along the ellipse, the contributions of mode 1 and 2 are changing such that the vortices coherently move along the ISL, as it would be expected for the precessing PVC helix. The relation between the mode coefficients given by Eq. 16 therefore provides a means to determine the phase angle $\varphi$ of the PVC precession, which is an important feature of the POD analysis. Having obtained the mode coefficients $a_{i 1}$ and $a_{i 2}$ according to Eq. 4, the phase angle $\varphi_{i}$ can be determined for each instantaneous velocity field $\vec{u}_{i}$ as

$\varphi_{i}=\frac{1}{\mathrm{i}} \ln \frac{a_{i 1}+\mathrm{i} a_{i 2}}{\sqrt{a_{i 1}^{2}+a_{i 2}^{2}}}$

In accordance with temporally resolved measurements of a PVC (Boxx et al. 2010), the sign of $\varphi$ in Eq. 17 was chosen such that vortices move downstream as $\varphi$ increases. Furthermore, for the vertical section the angle $\varphi=0^{\circ}$ was arbitrarily defined as $a_{i 1}>0$ and $a_{i 2}=0$. Subsequent to the estimation of the $\varphi_{i}$, phase-conditioned averages can be calculated for the velocity field and, if available, for other data measured at the same time.

Estimation of the phase angle of periodic vortex motion is often performed using local information from, e.g., pressure probes. In many cases, such local estimates are considerably affected by statistical fluctuations ("phase jitter"). By contrast, the estimation of the phase angle from the POD mode coefficients according to Eq. 17 implicitly takes into account the whole field of velocities and exclusively uses the coherent part of it. As it was found by van Oudheusden et al. (2005) and Perrin et al. (2007) for vortex shedding in cylinder wakes, such global phase indicators are significantly less affected by phase jitter and thus provide a more accurate estimate of the phase angle.

\subsection{Relation between vertical and horizontal sections}

Until now, no direct link between the observed zigzagordered vortices in the vertical section and the offcentered vortex seen in the horizontal sections has been established. It is therefore not yet sure if these two types of planar vortex structures both represent the same large-scale coherent vortex, namely the helical PVC. It is thus desirable to find a relation between the POD modes in the vertical and horizontal sections, which represent the two types of planar vortex structures. The only region where the POD modes can be compared is the line where the vertical and horizontal plane intersect. Representatively, a comparison of the vertical plane with the horizontal plane at $y=10 \mathrm{~mm}$ is made in the following (equivalent results were found for $y=5$ $\mathrm{mm}$ and $y=20 \mathrm{~mm}$ ). In this case, the velocity profiles along the intersection line $\{y=10 \mathrm{~mm}, z=0 \mathrm{~mm}\}$ in $\mathrm{x}-$ direction are considered. If the velocity profiles of the POD modes from both planes along this line are equal, this would indicate that the vortices in the vertical and horizontal plane correspond to a single $3 \mathrm{D}$ vortex structure.

Following Sects. 5.1 and 5.2, in each plane the flow related to the vortices can be represented by a linear superposition of the first two POD modes according to

$\vec{u}_{\text {vortex }}(\varphi)=\sqrt{2 \lambda_{1}} \cos \varphi \vec{\phi}_{1}+\sqrt{2 \lambda_{2}} \sin \varphi \vec{\phi}_{2}$,

where the phase angle $\varphi$ represents the varying vortex positions. For a meaningful comparison, the velocity profiles from the vertical and horizontal plane must be evaluated at the same phase angle. Since the measurements in each plane were performed independently, however, the zero phase angle $\varphi_{\text {vert }}=0^{\circ}$ for the vertical section does generally not correspond to $\varphi_{\text {horiz }}=0^{\circ}$ in the horizontal section. The appropriate value of $\varphi_{\text {horiz }}$ for the velocity profile of the horizontal section was therefore chosen such that the similarity to the profile of the vertical section at $\varphi_{\text {vert }}=0^{\circ}$ is highest. This was found to be the case at $\varphi_{\text {horiz }}=204^{\circ}$, and the phase angle of the horizontal section is corrected by this offset in the following. The resulting axial velocity profiles for $\varphi=0^{\circ}$ along the intersection line are plotted in Fig. 10. It can be seen that the velocity profiles from the vertical and from the horizontal section are in good agreement. A similar agreement was also found for the corresponding radial velocities and for other phase angles (not shown). It is therefore concluded that the POD mode pairs in the vertical and horizontal section represent the same $3 \mathrm{D}$ vortex structure, or in other words, that the vortices seen in the vertical and horizontal section are both manifestations of the helical PVC. 


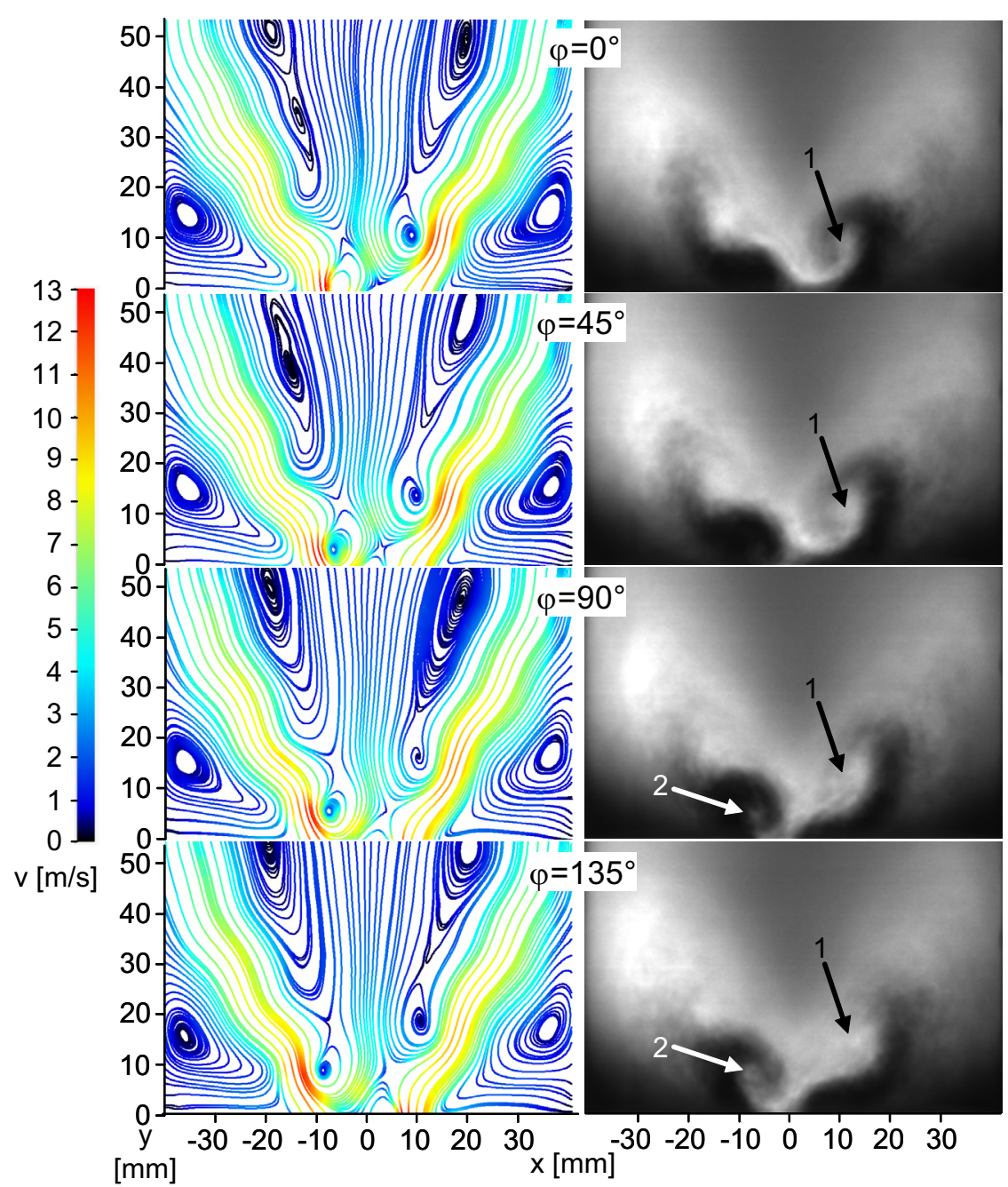

Fig. 11 Phase averages of velocity field (left) and $\mathrm{OH}$ distribution (right) in the vertical section. Colors represent the absolute value of velocity.

\section{Phase averages of velocity and $\mathrm{OH}$}

\subsection{General remarks}

The instantaneous PIV and OH-PLIF measurements discussed in Sect. 4.2 have already demonstrated the impact of the PVC on the flame. In the instantaneous velocity fields, however, the flow caused by the PVC is superimposed by turbulence and other unsteady dynamics (the PVC contributes only $27 \%$ to the velocity fluctuations in the reduced vertical section, cf. Sect. 5.1). Correspondingly, the instantaneous flame structures are affected by fluctuations of temperature and mixture fraction that are not directly related to the PVC. For a more rigorous analysis of vortex-flame interaction, the fields of velocity and $\mathrm{OH}$ have therefore been conditionally averaged with respect to the phase angle $\varphi$ of the PVC. In this way, all fluctuations that are not correlated to the phase of the PVC are cancelled out. For each of the 1500 instantaneous measurements, $\varphi_{i}$ was determined according to Eq. 17, and averages were then calculated for eight phases $\left(0^{\circ} \pm 22.5^{\circ}, 45^{\circ} \pm 22.5^{\circ}\right.$, $\left.90^{\circ} \pm 22.5^{\circ}, \ldots, 315^{\circ} \pm 22.5^{\circ}\right)$.

\subsection{Phase averages in planar sections}

The resulting phase averages of velocity and $\mathrm{OH}$ for the vertical section at $\varphi=0^{\circ}, 45^{\circ}, 90^{\circ}$ and $135^{\circ}$ are shown in Fig. 11. The structures for $\varphi=180^{\circ}, 225^{\circ}, 270^{\circ}$ and $315^{\circ}$ are equivalent but mirrored at the central axis $(x=0$ $\mathrm{mm})$. The velocity fields show the regular downstream motion of vortices in the ISL. In the $\mathrm{OH}$ images, arrow 1 tracks the position of a vortex. At $\varphi=0^{\circ}$, it can be seen that the vortex causes strong mixing of burned (light gray) and unburned gas (black). At $\varphi=45^{\circ}$, mixing continues and at the same time the $\mathrm{OH}$ level at the vortex 


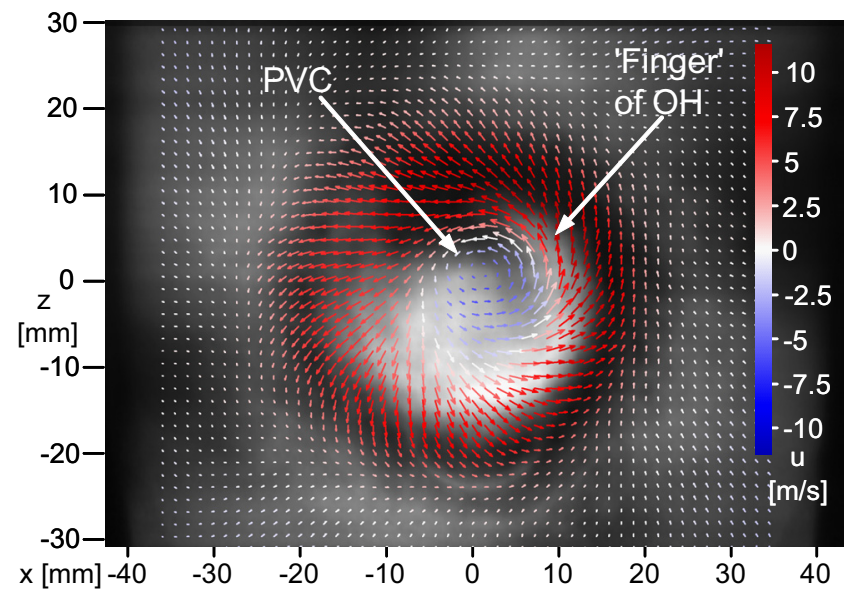

Fig. 12 Phase average of velocity field and $\mathrm{OH}$ distribution in the horizontal section at $y=10 \mathrm{~mm}$ for $\varphi=0$. Vector colors represent the axial velocity.

center increases, indicating that reaction takes place. The instantanteous measurement shown in Fig. 4 suggests that reaction rates in this region are enhanced by vortex-induced flame roll-up. At $\varphi=90^{\circ}$ and $\varphi=135^{\circ}$, further increase of $\mathrm{OH}$ levels around the vortex indicates reaction progress. At $\varphi=90^{\circ}$, one can further see the onset of reaction at the center of a vortex on the left side (arrow 2). The progress of reaction and the mixing induced by the vortex appear at $\varphi=135^{\circ}$.

Figure 12 shows phase averages of velocity and $\mathrm{OH}$ for $\varphi=0^{\circ}$ in the horizontal section at $y=10 \mathrm{~mm}$. It can be seen that the PVC induces a finger-like structure of $\mathrm{OH}$ in the ISL. At other phase angles (not shown) the same structure appears but rotated counterclockwise around the centerline $(x=z=0 \mathrm{~mm})$. Accordingly, at $\varphi=90^{\circ}$ the finger of $\mathrm{OH}$ starts to penetrate through the vertical section $(z=0 \mathrm{~mm})$. In the phase averages of the vertical section discussed above, this corresponds to the appearance of $\mathrm{OH}$ at the vortex center on the left side at $\varphi=90^{\circ}$. It is thus concluded that a significant part of heat release takes place in a tubular structure that is located along the PVC and rotates around the centerline.

\subsection{Determination of 3D structure}

Previous studies have shown that the PVC is a $3 \mathrm{D}$ helical vortex structure that precesses around the central axis (Syred 2006). It is therefore possible to determine the 3D structure of the PVC by using the phase angle $\varphi$ as the azimuth of a cylindrical coordinate system. This approach is pursued in the following. At first, the swirling strength has been calculated for the instantaneous $2 \mathrm{D}$ velocity fields in order to determine the pres- ence of vortices (Adrian et al. 2000). Phase averages of the swirling strength were then calculated and are shown in Fig. 13a for $\varphi=0^{\circ}, 45^{\circ}, 90^{\circ}$ and $135^{\circ}$. It can be seen that vortices do not only appear in the ISL, but with lower strength also in the OSL. Such vortices in the OSL have already been seen in the instantaneous velocity field shown in Fig. 4a (gray arrows). The fact that these vortices appear in the phase averages verifies that they are coupled to the PVC in the ISL. The corresponding 3D structure of swirling strength is shown as an isosurface plot in Fig. 13b. It shows that the PVC helix makes one full revolution around the central axis within an axial distance of $y=15 \mathrm{~mm}$. The helical vortex in the OSL is located along the PVC. The influence of the outer vortex on the flame is rather small because most part of the reaction takes place in the ISL (cf. Figs. 3 and 4).

\subsection{Phase averaging vs low-order modelling}

Until now vortex-flame interaction has been studied using fields of velocity and $\mathrm{OH}$ that have been conditionally averaged with respect to the phase angle of the PVC. An alternative approach for studying this interaction would be the use of an extended POD analysis. As described in Sect. 3.3, the extended POD mode $\chi_{j}$ relates to the part of the $\mathrm{OH}$ field that is correlated with the corresponding velocity POD mode $\vec{\phi}_{j}$. The effect of the PVC on the flame can then be determined by low-order descriptions of both the velocity field and the $\mathrm{OH}$ field that are restricted to the first two modes representing the PVC. As described in Sect. 3, a loworder description can be determined for each instantaneous field of velocity and $\mathrm{OH}$ by using the corresponding mode coefficients $a_{i 1}$ and $a_{i 2}$. In the following, a low-order model is determined that is a function of the phase angle $\varphi$. According to Eqs. 16 and 17, the phasedependent coefficients for mode 1 and 2 can be written as

$a_{\varphi 1}=\sqrt{2 \lambda_{1}} \cos \varphi$,

$a_{\varphi 2}=\sqrt{2 \lambda_{2}} \sin \varphi$.

The low-order model of the velocity field is then given by

$\vec{u}_{\mathrm{LO}}(\varphi)=\overline{\vec{u}}+a_{\varphi 1} \vec{\phi}_{1}+a_{\varphi 2} \vec{\phi}_{2}$.

In an analogous way, the extended low-order model for the $\mathrm{OH}$ field is given by

$c_{\mathrm{LO}}(\varphi)=\bar{c}+a_{\varphi 1} \chi_{1}+a_{\varphi 2} \chi_{2}$.

As discussed in Sect. 3.3, $c_{\mathrm{LO}}(\varphi)$ is exactly that part of the concentration field that is correlated to $\vec{u}_{\mathrm{LO}}(\varphi)$. 

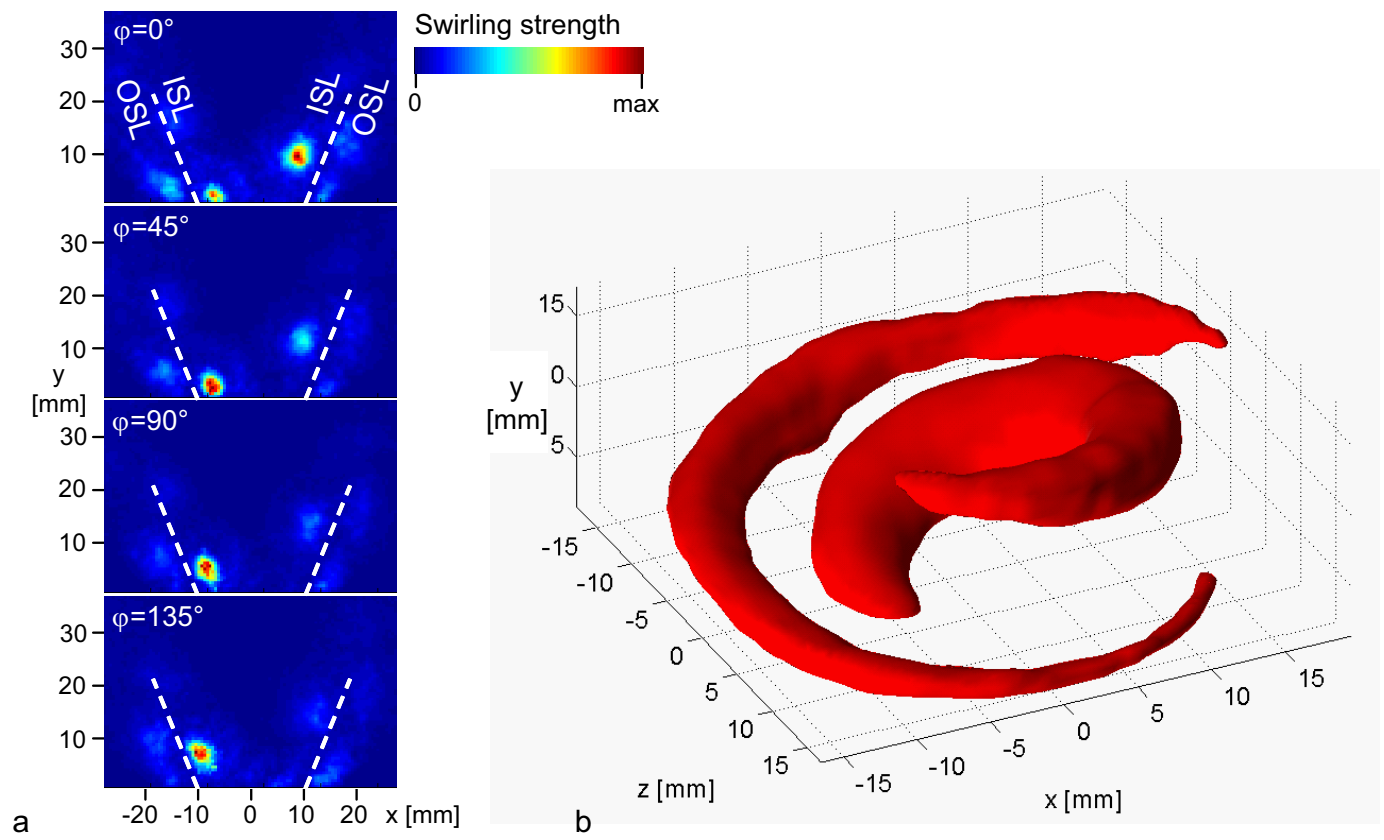

Fig. 13 (a) 2D phase averages and (b) 3D structure of swirling strength.

In Fig. 14a,b, $c_{\mathrm{LO}}\left(\varphi=135^{\circ}\right)$ is compared to the corresponding phase average. Although the patterns of the $\mathrm{OH}$ field are largely similar, the structures generally appear more pronounced in the phase average than in the low-order model. E.g., the twisted structure on the left side (arrow 1) and the boundary between burned and unburned gas (arrow 2) are more well marked in the phase average. Similar effects can be seen in the comparison of the low-order velocity field $\vec{u}_{\mathrm{LO}}\left(\varphi=45^{\circ}\right)$ (Fig. 14d) and the corresponding phase average (Fig. 14c). Here, e.g., the vortex structure on the lower left side (arrow 3 ) is clearly more pronounced in the phase average.

A comparison of phase averages and a POD-based second-order model has been made previously in the context of periodic vortex shedding in cylinder wakes by van Oudheusden et al. (2005). Like this work, the study found the effect that the spatial structures in the second-order model are smooth compared to the phase averages. It was further shown that in the loworder model, variation of velocity with respect to $\varphi$ is restricted to a sinusoidal shape, and that the spatial smoothing results from this restriction. The same restriction applies to the low-order model of velocity and $\mathrm{OH}$ in the present study. Fig. 15 shows the phasedependent variation of $\mathrm{OH}$ at $x=-9.4 \mathrm{~mm}, y=4.4 \mathrm{~mm}$. It can be seen that the low-order model exhibts a sinusoidal shape and cannot follow the rapid increase and decay of $\mathrm{OH}$ around $\varphi=225^{\circ}$ that takes place in the phase averages.
The augmented dynamics seen in the phase averages results from the different ways of conditioning. Whereas the phase averages are conditioned on a single quantity, namely the phase angle $\varphi$, the low-order representation is conditioned on the first two POD modes, i.e., on the whole field of velocities. The phase averages therefore additionally take into account transient variations of the shape of the PVC that are not represented in the first two POD modes.

In cases where only a small number (say, 50 or less) of instantaneous measurements are available per phase angle, phase averages are considerably affected by noise due to insufficient statistical convergence. These statistical fluctuations are significantly lowered in the corresponding low-order representation due to the restrictions discussed above, and therefore it may be preferable to use the low-order representation for further analysis in such situations. If, by contrast, the number of samples is high enough such that statistical fluctuations in the phase averages are sufficiently low, the above results show that phase averages are more useful than the low-order model because they can capture variations that are not necessarily sinusoidal.

\section{Summary and conclusions}

The relation between flow field and flame structure of a turbulent swirl flame in a gas turbine model combustor has been investigated using simultaneous particle image velocimetry (PIV) and planar laser-induced fluo- 


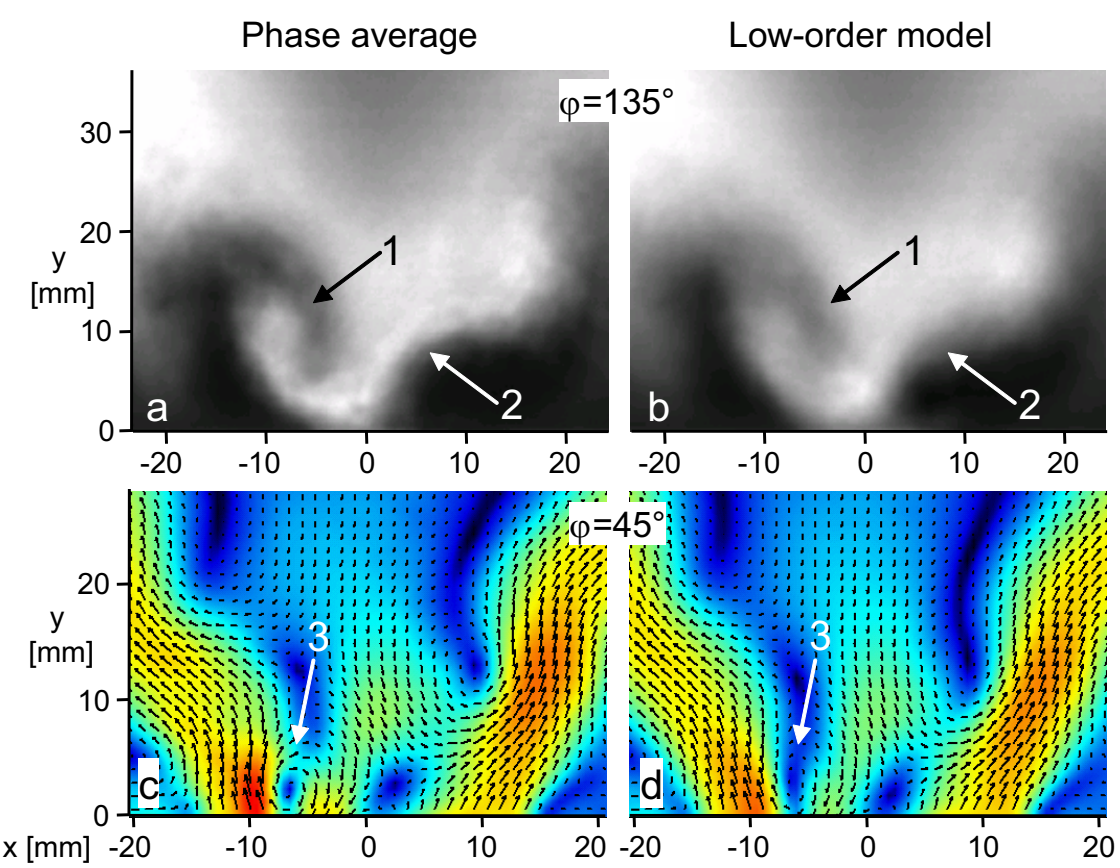

Fig. 14 Comparison of phase averages (left) and the corresponding low-order model (right), shown for OH distribution at $\varphi=135^{\circ}$ (top) and for the velocity field at $\varphi=45^{\circ}$ (bottom).

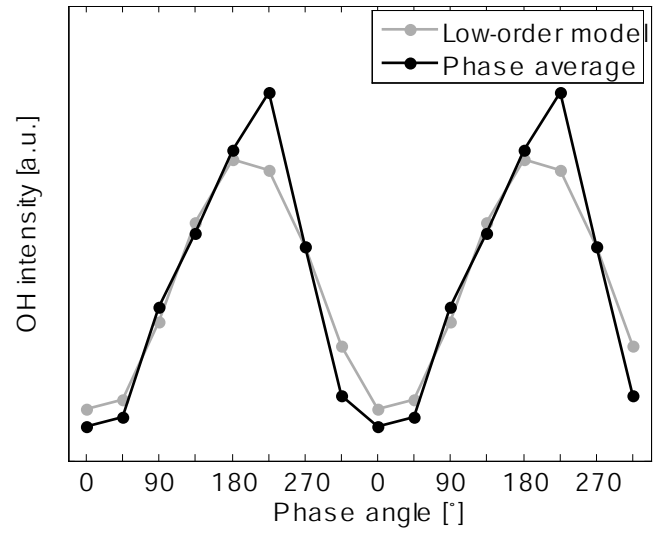

Fig. 15 Variation of $\mathrm{OH}$ intensity with phase angle in the phase averages and in the low-order model.

rescence (PLIF) of OH. The measured average flow field and flame shape have shown that the flame is stabilized mainly by an inner recirculation zone which transports hot reaction products back to the flame base, which is typical for confined swirl flames. The instantaneous velocity fields showed a zig-zag arrangement of vortices in the inner shear layer, which indicates the presence of a helical precessing vortex core (PVC). The OH-PLIF images showed that the PVC is located at the boundary between the stream of fresh gas and the recirculating burned gas, where a large part of heat release takes place.
A particular focus of this work was the application of proper orthogonal decomposition (POD) for the characterization of coherent flow structures and their interaction with the flame. A POD of the velocity field showed that the PVC is the dominant unsteady flow structure, extending with decreasing strength from the nozzle up to a height of $y \approx 40 \mathrm{~mm}$. The phase angle of the precession could be accurately determined for each instantaneous velocity field from its projection on the first two POD modes representing the PVC. Thereby, phase-conditioned averages of velocity field and $\mathrm{OH}$ distribution were obtained and revealed a pronounced effect of the PVC in the form of convection-enhanced mixing. It was seen that the increased mixing causes a rapid ignition of the fresh gas and that the swirling motion of the PVC leads to an enlarged flame surface due to flame roll-up. Using the phase angle as the azimuth of cylindrical coordinates, the three-dimensional structure of PVC has been determined. This representation showed that the PVC is accompanied by a coprecessing vortex in the outer shear layer, which, however, has no direct impact on the flame. As an alternative to phase averaging, a low-order representation of the phase-resolved dynamics has been calculated based on the first pair of POD modes. It was found that smallscale structures are represented more accurately in the phase averages, whereas the low-order model has a considerable smoothing effect and therefore provides less detailed information. The findings demonstrate that the 
combined application of POD, PIV and PLIF can provide detailed insights into flow-flame interaction in turbulent flames.

\section{References}

Adrian RJ, Christensen KT, Liu ZC (2000) Analysis and interpretation of instantaneous turbulent velocity fields. Exp Fluids 29: 275-290

Berkooz G, Holmes P, Lumley JL (1993) The proper orthogonal decomposition in the analysis of turbulent flows. Annu Rev Fluid Mech 25:539-575

Böhm B, Heeger C, Boxx I, Meier W, Dreizler A (2009) Time-resolved conditional flow field statistics in extinguishing turbulent opposed jet flames using simultaneous high-speed PIV/OH PLIF. Proc Combust Inst 32: 1647-1654

Boxx I, Stöhr M, Carter C, Meier W (2010) Temporally resolved planar measurements of transient phenomena in a partially pre-mixed swirl flame in a gas turbine model combustor. Combust Flame 157: 15101525

Borée J (2003) Extended proper orthogonal decomposition: a tool to analyse correlated events in turbulent flows. Exp Fluids 35: 188-192

Chaudhuri S, Kostka S, Renfro MW, Cetegen BM (2010) Blowoff dynamics of bluff body stabilized turbulent premixed flames. Combust Flame 157: 790-802

Chaudhuri S, Kostka S, Tuttle SG, Renfro MW, Cetegen BM (2011) Blowoff mechanism of two dimensional bluff-body stabilized turbulent premixed flames in a prototypical combustor. Combust Flame, in press

Duwig C, Iudiciani P (2010) Extended proper orthogonal decomposition for analysis of unsteady flames. Flow Turbulence Combust (2010) 84: 25-47

Hardalupas Y, Orain M (2004) Local measurements of the time-dependent heat release rate and equivalence ratio using chemiluminescent emission from a flame. Combust Flame 139: 188-207

Hult J, Meier U, Meier W, Harvey A, Kaminski CF (2005) Experimental analysis of local flame extinction in a turbulent jet diffusion flame by high repetition 2D laser techniques and multi-scalar measurements. Proc Combust Inst 30: 701-709

Konle M, Kiesewetter F, Sattelmayer T (2008) Simultaneous high repetition rate PIVLIF-measurements of CIVB driven flashback. Exp Fluids 44: 529-538

Konle M, Sattelmayer T (2009) Interaction of heat release and vortex breakdown during flame flashback driven by combustion induced vortex breakdown. Exp Fluids 47: 627-635
Kothnur PS, Tsurikov MS, Clemens NT, Donbar JM, Carter CD (2002) Planar imaging of $\mathrm{CH}, \mathrm{OH}$, and velocity in turbulent non-premixed jet flames. Proc Combust Inst 29: 1921-1927

Lemaire A, Meyer TR, Zähringer K, Gord JR, Rolon JC (2004) PIV/PLIF investigation of two-phase vortexflame interactions: effects of vortex size and strength. Exp Fluids 36: 36-42

Lumley JL (1967) The structure of inhomogeneous turbulence. In: Yaglom AM, Tatarski VI (eds) Atmospheric turbulence and wave propagation. Nauka, Moscow, 166-178

Meier W, Boxx I, Stöhr M, Carter CD (2010) Laserbased investigations in gas turbine model combustors. Exp Fluids 49: 865-882

Meyer TR, Fiechtner GJ, Gogineni SP, Rolon JC, Carter CD, Gord JR (2004) Simultaneous PLIF/PIV investigation of vortex-induced annular extinction in $\mathrm{H}_{2}$-air counterflow diffusion flames. Exp Fluids 36: 259-267

Mueller CJ, Driscoll JF, Sutkus DJ, Roberts WL, Drake MC, Smooke MD (1995) Effect of unsteady stretch rate on $\mathrm{OH}$ chemistry during a flame-vortex interaction to assess flamelet models. Combust Flame 100: 323-331

Mueller CJ, Driscoll JF, Reuss DL, Drake MC, Rosalik ME (1998) Vorticity generation and attenuation as vortices convect through a premixed flame. Combust Flame 112: 342-346

Perrin R, Braza M, Cid E, Cazin S, Barthet A, Sevrain A, Mockett C, Thiele F (2007) Obtaining phase averaged turbulence properties in the near wake of a circular cylinder at high Reynolds number using POD. Exp Fluids 43: 341-355

Petersson P, Olofsson J, Brackman C, Seyfried H, Zetterberg J, Richter M, Aldén M, Linne MA, Cheng RK, Nauert A, Geyer D, Dreizler A (2007) Simultaneous PIV/OH-PLIF, Rayleigh thermometry/OHPLIF and stereo PIV measurements in a low-swirl flame. Appl Opt 46: 3928-3936

Rehm JE, Clemens NT (1998) The relationship between vorticity/strain and reaction zone structure in turbulent nonpremixed jet flames. Proc Comb Inst 27: 1113-1120

Renard PH, Rolon JC, Thévenin D, Candel S (1999) Investigations of heat release, extinction, and time evolution of the flame surface, for a nonpremixed flame interacting with a vortex. Combust Flame 117:189205

Renard PH, Thévenin D, Rolon JC, Candel S (2000) Dynamics of flame/vortex interactions. Prog Energy Combust Sci 26: 225-282 
Sadanandan R, Stöhr M, Meier W (2008) Simultaneous OH-PLIF and PIV measurements in a gas turbine model combustor. Appl Phys B 90: 609-618

Sirovich L (1987) Turbulence and the dynamics of coherent structures. Quart Appl Math 45: 561-590

Steinberg AM, Boxx I, Stöhr M, Carter CD, Meier W (2010) Flow-flame interactions causing acoustically coupled heat release fluctuations in a thermoacoustically unstable gas turbine model combustor. Combust Flame 157: 2250-2266

Stöhr M, Sadanandan R, Meier W (2009) Experimental study of unsteady flame structures of an oscillating swirl flame in a gas turbine model combustor. Proc Combust Inst 32: 2925-2932

Stöhr M, Boxx I, Carter C, Meier W (2011) Dynamics of lean blowout of a swirl-stabilized flame in a gas turbine model combustor. Proc Combust Inst 33: 2953-2960

Syred N (2006) A review of oscillation mechanisms and the role of the precessing vortex core (PVC) in swirl combustion systems. Prog Energy Combust Sci 32: 93-161

Tanahashi M, Muratami S, Choi G-M, Fukuchi Y, Miyauchi T (2005) Simultaneous CH-OH PLIF and stereoscopic PIV measurements of turbulent premixed flames. Proc Combust Inst 30: 1665-1672

van Oudheusden BW, Scarano F, van Hinsberg NP, Watt DW (2005) Phase-resolved characterization of vortex shedding in the near wake of a square-section cylinder at incidence. Exp Fluids 39: 86-98

Watson KA, Lyons KM, Carter CD, Donbar JM (2002) Simultaneous two-shot $\mathrm{CH}$ planar laser-induced fluorescence and particle image velocimetry measurements in lifted $\mathrm{CH}_{4} /$ air diffusion flames. Proc Combust Inst 29: 1905-1912

Weigand P, Meier W, Duan XR, Stricker W, Aigner M (2006) Investigations of swirl flames in a gas turbine model combustor I. Flow field, structures, temperature, and species distributions. Combust Flame 144: 205-224

Wicksall DM, Agrawal AK, Schefer RW, Keller JO (2005) The interaction of flame and flow field in a lean premixed swirl-stabilized combustor operated on $\mathrm{H}_{2} / \mathrm{CH}_{4}$ /air. Proc Combust Inst 30: 2875-2883 